\title{
A Method of Calculating Tunneling Corrections For Eckart Potential Barriers
}

\author{
R. L. Brown* \\ National Bureau of Standards, Washington, DC 20234 \\ February 18, 1981
}

\begin{abstract}
A method is presented for the direct calculation of tunneling corrections for unsymmetrical Eckart type potential barriers. It is based on a modified 6-point Gaussian quadrature formula. Accuracy is better than 1 percent over a wide range of tunneling parameter values.
\end{abstract}

Key words: chemical rate constants; Eckart potential; potential barriers; quantum mechanical tunneling calculations; tunneling corrections.

\section{Introduction}

The Eckart potential function [1] ${ }^{1}$ is often used to estimate quantum mechanical tunneling corrections to theoretically determined chemical rate constants. The correction factor $\Gamma^{*}$ is defined as the ratio of the quantum mechanical to the classical mechanical barrier crossing rate. It can be expressed [2] as in integral over the energy $E$,

$$
\Gamma^{*}=\exp \left(V_{1} / k T\right) \int_{\mathrm{E}_{\odot}}^{\infty} K \exp (-E / k T) d E / k T
$$

where $V_{1}$ is the height of the potential barrier, and $K$ is the transmission probability for tunneling. $K$ depends on $E$ and three other parameters which are determined by the shape of the barrier and an effective mass for the system. Johnston and Heicklen [3] have evaluated this integral numerically for a number of parameter values. For certain applications their results are inconvenient to use because interpolation is required to get values not tabulated. In view of this, I have devised a simple method which can be used to calculate $\Gamma^{*}$ directly, for any set of parameter values within the ranges chosen by Johnston and Heicklen. The method is presented in the form of a small FORTRAN subroutine called TUNL. In the next section, the details of the method are discussed. Following this, the results of a series of comparisons with an accurate calculation are presented. Finally, the subroutine is listed in the Appendix.

*Center for Thermodynamics and Molecular Science, National Measurement Laboratory.

\section{Derivation of the method}

Eckart's potential has the form

$$
\begin{aligned}
& V=-y[A-B /(1-y)] /(1-y) \\
& y=-\exp (2 \pi x / L) \\
& A=V_{1}-V_{2} \\
& B=\left(V_{1}^{1 / 2}+V_{2}^{1 / 2}\right)^{2} \\
& L=2 \pi\left(-2 / F^{*}\right)^{1 / 2}\left(V_{1}^{-1 / 2}+V_{2}^{-1 / 2}\right)^{-1}
\end{aligned}
$$

The potential has the limiting value of zero when $x \rightarrow-\infty$, goes through a single maximum of height $V_{1}$ as $x$ increases, and has a limiting value of $V_{1}-V_{2}$ as $x \rightarrow+\infty$. $F^{*}$ is the second derivative of $V$ at its maximum. The lower bound $E_{0}$ in the integral (1) is equal to zero when $V_{1} \leq V_{2}$, and to $V_{1}$ $-V_{2}$ when $V_{1}>V_{2}$. The three parameters used by Johnston and Heicklen are $\alpha_{1}, \alpha_{2}$, and $u^{*}$.

$$
\begin{aligned}
u^{*} & =h \nu^{*} / k T \\
\alpha_{i} & =2 \pi V_{i} / h \nu^{*}, i=1,2 \\
\nu^{*} & =(1 / 2 \pi)\left(-F^{*} / m\right)^{1 / 2}
\end{aligned}
$$

where $m$ is an effective mass for tunneling (see ref. 2, p. 53). The integral (1) can be written in a symmetrical form by introducing a new variable, $\epsilon=\left(E-V_{1}\right) / k T$. It becomes

$$
\Gamma^{*}=\int_{\epsilon_{0}}^{\infty} K e^{-\epsilon} d \epsilon
$$

where $\epsilon_{0}=-v_{1}=-V_{1} / k T$ when $V_{1} \leq V_{2}$, and $\epsilon_{o}=-v_{2}$ $=-V_{2} \mid k T$ when $V_{1}>V_{2}$. In terms of the parameters (2), 
the transmission probability $K$, derived by Eckart, has the form

$$
\begin{aligned}
& K=\left[\cosh 2 \pi\left(a_{1}+a_{2}\right)-\cosh 2 \pi\left(a_{1}-a_{2}\right)\right] \times \\
& {\left[\cosh 2 \pi\left(a_{1}+a_{2}\right)+D\right]^{-1} } \\
& 2 \pi a_{i}=\pi\left[\left(\epsilon+v_{i}\right) / c\right]^{1 / 2}, i=1,2 \\
& c=(1 / 8) \pi u^{*}\left(\alpha_{1}{ }^{-1 / 2}+\alpha_{2}^{-1 / 2}\right)^{2} \\
& D=\cosh 2 \pi d \text { if } d \text { is real } \\
&=\cos 2 \pi|d| \text { if } d \text { is imaginary } \\
& 2 \pi d=\left(4 \alpha_{1} \alpha_{2}-\pi^{2}\right)^{1 / 2}
\end{aligned}
$$

The method used to evaluate (3) is a modified 6-point Gaussian quadrature formula based on Legendre polynomials [4]. This was used even though the nature of the integral suggests using a formula based on Laguerre polynomials. When the number of evaluations of $K$ is kept small, neither of these methods is satisfactory for the whole range of parameter values used by Johnston and Heicklen, so a variation of the first method was developed.

When $\epsilon$ gets large, $K$ approaches unity. The method uses a Gaussian formula for that part of the integral where $K<$ 1. The remainder where $K \approx 1$ is evaluated analytically. Thus, if $K(\epsilon) \approx 1$ for $\epsilon>\epsilon_{b}$, then

$$
\int_{\epsilon_{0}}^{\infty} K(\epsilon) e^{-t} d \epsilon \approx \int_{\epsilon_{0}}^{\infty} e^{-\epsilon} d \epsilon=e^{-\epsilon} .
$$

To evaluate $\epsilon_{b}$, examine (4) as $\epsilon \rightarrow \infty$. One gets $\alpha_{i} \rightarrow$ $1 / 2(\epsilon / c)^{1 / 2}$ and

$$
K-1-(1+D)\left(1 / 2 \exp \left(2 \pi\left(\epsilon_{b} / c\right)^{1 / 2}\right)+D\right)^{-1}=K_{b} .
$$

Setting $K_{b}$ to some value close to unity and solving this equation for $\epsilon_{b}$ gives

$$
\epsilon_{b}=c\left\{\frac{1}{2 \pi} \log \left[\frac{2(1+D)}{1-K_{b}}\right]\right\}^{2}
$$

It happens that this value is not entirely satisfactory, and subtracting from it the average value of $v_{1}$ and $v_{2}$ gives better results. Also, in some cases, $\epsilon_{b}$ calculated in this way is very large. There is no point in using this value for $\epsilon_{b}$ as the upper bound of the Gaussian formula if the integrand at this point is negligible because of the exponential factor. Thus $\epsilon_{b}$ was kept below a certain fixed value $\epsilon_{\max }$. There resulted two parameters, $K_{b}$ and $\epsilon_{\max }$, which were adjusted to minimize the sum of the squares of the differences between the results of this method and the corresponding tabulated values of Johnston and Heicklen.

\section{Test of the method}

Extensive testing of the accuracy of the method was performed by comparing it with an accurate 40-point Gaussian formula having the cutoff fixed at $\epsilon_{b}$ corresponding to $K_{b}=$ 0.999 or at $\epsilon_{b}<8$. In the ranges $0.5 \leq \alpha_{1} \leq \alpha_{2} \leq 20$, and $2 \leq u^{*} \leq 16$, a group of 10,910 comparisons was made. For this set there were the additional restrictions that when $\alpha_{1}$ $\geq 8$ then $u^{*} \leq 12$, or when $\alpha_{1} \geq 16$ then $u^{*} \leq 10$. Note that $\Gamma^{*}\left(\alpha_{1}, \alpha_{2}\right)=\Gamma^{*}\left(\alpha_{2}, \alpha_{1}\right)$. A second set of 4,920 comparisons was made in the ranges $0.5 \leq \alpha_{1} \leq \alpha_{2} \leq 20$, and 0.05 $\leq u^{*} \leq 1.5$. The results of these tests are given in table 1 in the form of histograms. These show the number of values which differ from the accurate values by a given percentage range. It can be seen from these results that very few values are in error by as much as $\mathbf{5}$ percent. Such accuracy should be quite adequate for most rate constant calculations.

TABLE I. Tests of the accuracy of TUNL

\begin{tabular}{l|c|c}
\hline $\begin{array}{r}\text { Variation from } \\
\text { accurate values }\end{array}$ & \multicolumn{2}{c}{$\begin{array}{c}\text { Number of differences in } \\
\text { percentage ranges }\end{array}$} \\
\hline Percent difference & Set I ${ }^{a . b}$ & Set II $^{\text {a. c }}$ \\
\hline$-5.5 \%,-4.5 \%$ & 3 & 1 \\
$-4.5,-3.5$ & 4 & 5 \\
$-3.5,-2.5$ & 6 & 19 \\
$-2.5,-1.5$ & 30 & 26 \\
$-1.5,-0.5$ & 3246 & 140 \\
$-0.5,0.5$ & 6811 & 4475 \\
$0.5,1.5$ & 343 & 227 \\
$1.5,2.5$ & 217 & 27 \\
$2.5,3.5$ & 170 & \\
$3.5,4.5$ & 78 & \\
$4.5,5.5$ & 2 & $0.42 \%$ \\
\hline Standard deviations & $0.77 \%$ & \\
\hline
\end{tabular}

- For both Sets I and II, $\alpha_{2} \geq \alpha_{1}$. The values used were $0.5,1.0,1.5,2.0$, . 40.0.

${ }^{b}$ For Set $\mathrm{I}, u^{*}=2,3,4, \ldots 16$. Also if $\alpha_{1} \geq 8$ then $u^{*} \leq 12$ and if $\alpha_{1} \geq$ 16 then $u^{*} \leq 10$.

'For Set II, $u^{*}=0.05,0.1,0.2,0.5,1.0,1.5$.

\section{Appendix. Listing of TUNL}

The parameters for this program are ALPH1 $=\alpha_{1}$, ALPH2 $=\alpha_{2}, U=u^{*}$, and $G=\Gamma^{*}$. It will calculate $\Gamma^{*}$ accurately in the parameter ranges $0.5 \leq \alpha_{1}, \alpha_{2} \leq 20$, and 0 $<u^{*} \leq 16$ with the additional restrictions that when $\alpha_{1}$ and $\alpha_{2} \geq 8$ then $u^{*} \leq 12$, and when $\alpha_{1}$ and $\alpha_{2} \geq 16$ then $u^{*} \leq 10$. 
SUBROUTINE TUNL (ALPH , ALPH 2, U, G)

DIMENSION T(6), 16$)$ (6)

DATA PIOPI COSH $(Z)=S^{\circ}$ i EXP $\left.(Z)+E X P(-Z)\right\}$

$C=.125 * P I * U *(1 . / \operatorname{SQRT}(A L P H 1)+1, / \operatorname{SQRT}(A L P H 2)) \cdots 2$

$\mathrm{V} 1=\mathrm{UPI} 2: A L P H 1$

$D=4 \cdot A L P H 1 * A L P H 2-P I S Q$

DF = COSH i SQRT (D))

$10 \mathrm{DF}=\cos (\operatorname{SQRT}(-\mathrm{D}))$

IF (V2.GE. V1) EZ=-V1

$E B=A M I N 1(C *(A L O G(2, *(1,+D F) / .014) / P I 2) * 2-.5 *(V 1+V 2), 3.2)$ $E M=: 5 *(E B-E Z)$

$\mathrm{DO} 20 \mathrm{~N}=1,6$

$\mathrm{D} O$
$\mathrm{E}=\mathrm{EM}$

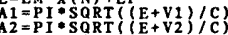

$20 \begin{aligned} & F M=C O S H \\ & G=G+P 1\end{aligned}$

G=EM $+E X P(-E B) \cdot(F P-F M) /(F P+D F)$

RETURN

\section{References}

[1] Eckart, Carl. The penetration of a potential barrier by electrons. Phys. Rev. 35(11): 1303-1309; 1930 June 1.

[2] Johnston, Harold S. Gas phase reaction rate theory. New York: Ronald Press; 1966, 362 p.

[3] Johnston, Harold S.; Heicklen, Julian. Tunneling corrections for unsymmetrical Eckart potential energy barriers. J. Phys. Chem. 66(3): 532-533; 1962 March.

[4] Abramowitz, M.; Stegun, I. A. Handbook of mathematical functions. Nat. Bur. Stand. (U.S.) Appl. Math. Ser. 55; 1964 June. 1045 p. 